
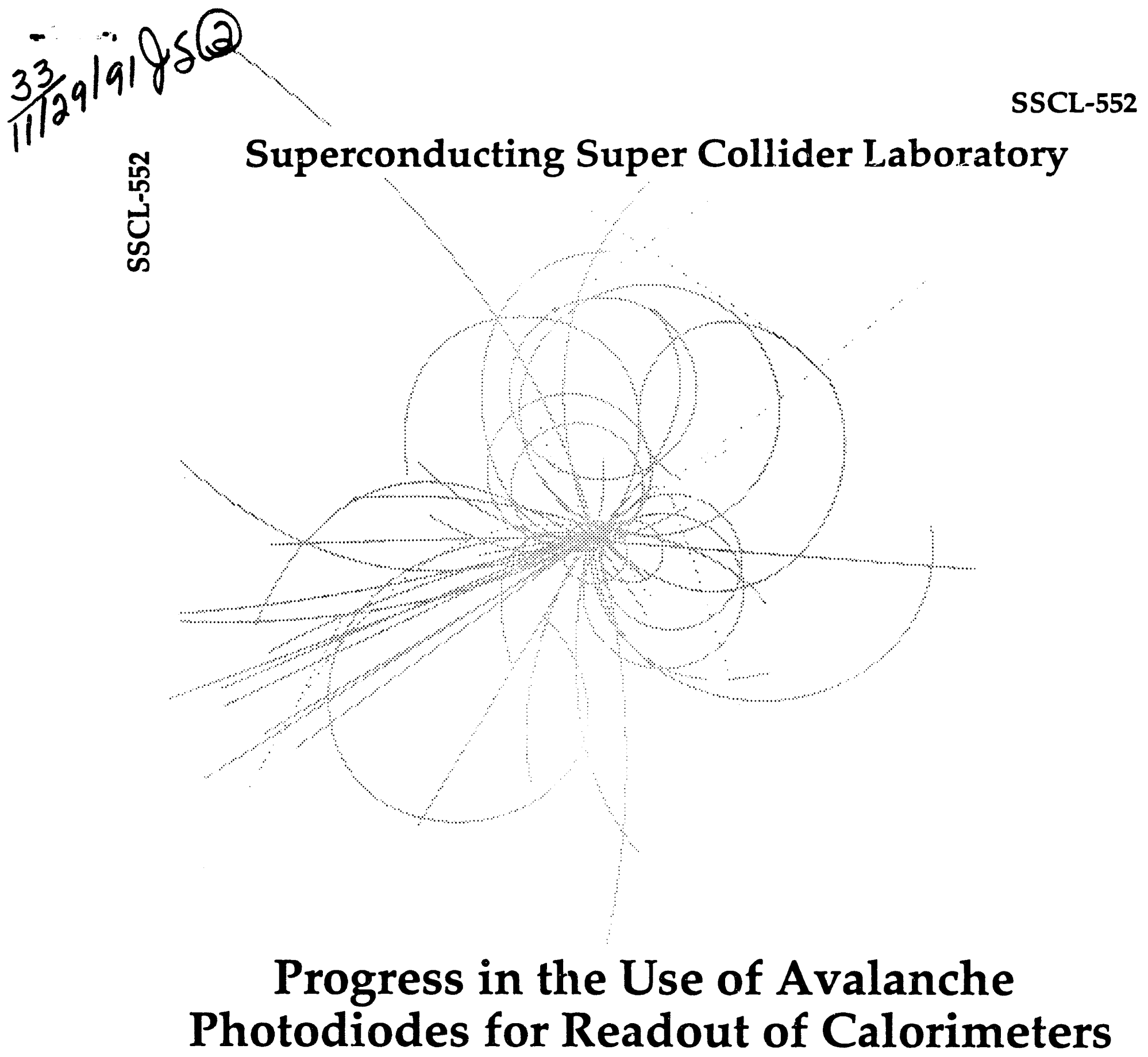

H. Fenker, K. Morgan, and T. Regan

September 1991 


\section{Progress in the Use of Avalanche Photodiodes for Readout of Calorimeters}

H. Fenker, K. Morgan, and T. Regan

Physics Research Division

Superconducting Super Collider Laboratory ${ }^{\dagger}$

2550 Beckleymeade Ave.

Dallas, TX 75237

September 1991

TOperated by the Universities Research Association, Inc., for the U.S. Department of Energy under Contract No. DE-AC35-89ER40486. 


\section{CONTENTS}

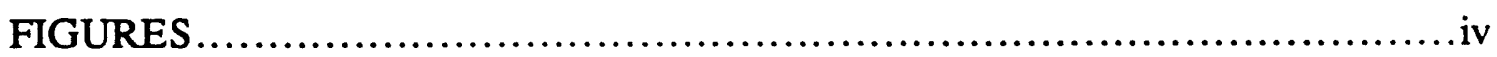

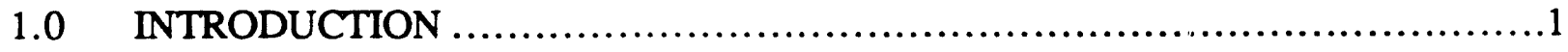

2.0 GENERAL CHARACTERIZATION OF APD OPERATION $\ldots \ldots \ldots \ldots \ldots \ldots \ldots \ldots \ldots . . \ldots \ldots$

3.0 SENSITIVE ELEMENT REQUIREMENTS FOR PRE-SHOWER/SHOWER-

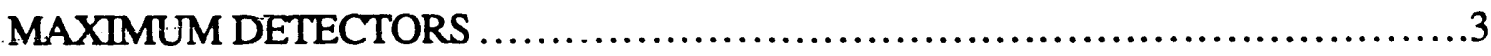

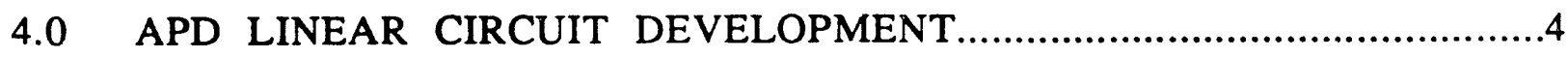

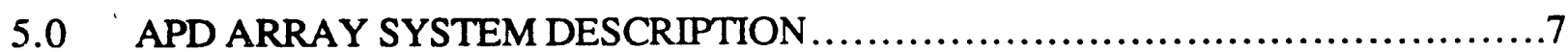

5.1 Electronic ............................................................

5.2 Mechanical............................................................. 12

5.3 Optical............................................................... 15

5.4 System Test Results................................................... 18

$6.0 \quad$ SUMMARY Y............................................................ 19

ACKNOWLEDGEMENTS ............................................... 21

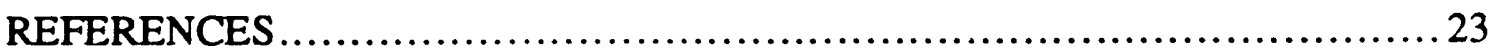




\section{FIGURES}

1. Spectral response curves for two commercial APDs................................

2. Oscilloscope trace showing the signal from an avalanche in an APD operated in Geiger mode. The same trace is shown at two different time scales. For this

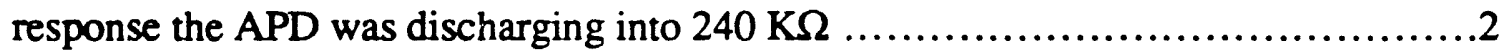

3. Test circuit used for observing Geiger mode APD signals.............................

4. The DO charge-sensitive preamp circuit used in initial linear mode tests of APDs......4

5. Block diagram of experimental arrangement for initial APD studies...................5

6. Early test results of an APD operating in linear mode and exposed to about 60 green $(565 \mathrm{~nm})$ photons. The electronic noise apparent in this figure was later reduced. .....5

7. Response of an APD to varying amounts of incident light. The scatter of the data above and below the straight line was reduced by stabilizing the temperature of the

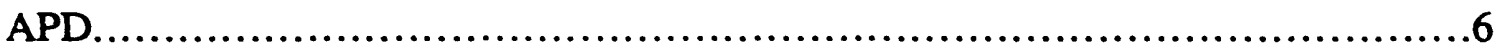

8. Variation of APD response with time of day. Thermal stabilization of the APD eliminated this variation. Note that error bars indicate the statistical spread in the signals. The error on the mean is of order one ADC count.................................

9a. Charge-sensitive amplifier circuit implemented in the 96-channel APD system. ........8

9b. Schematic diagram of a 16-channel APD/Amplifier/Driver circuit board. The three blocks at the top are the temperature monitoring circuits. ............................9

10 Photograph of the 96-channel array composed of six cards of 16 channels each. One card is shown with the RF shield removed. The APDs are imbedded in the bar at the front of each card. Optical connectors, one with fiber attached, are shown inserted in the topmost card. Thermal stability is provided by circulating water through the pipe attached to the front of the crate.

11. The Pre-shower Detector. Scintillating fiber ribbons are in the middle and are read out by various test devices on either end. The entire assembly is enclosed in a light-tight box.

12. Detail of the assembly of the electronic circuit board to the cooling system and crate. The avalanche photodiodes are mounted in the "isobar.".

13. The Feed-Through Panel. Constructed of copper-clad black substrate, this panel provides light-tight bulkheads for eight ribbon cables, four gas or water lines, eight BNC cables, four SHV cables, and a multiconductor low-voltage connector. ........ 14

14. Optical Alignment Procedure. The APD is mounted in a thermally stabilized brass

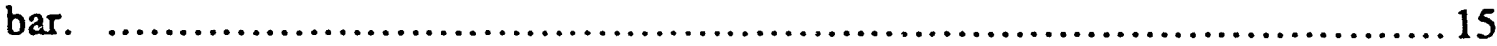

15. Cross section of the APD Photodiode developed by EG\&G Optoelectronics for use with $1-\mathrm{mm}$-diameter fibers. The APD itself is only $0.5 \mathrm{~mm}$ wide 
16. Map of the response of the APD with and without the ball lens assembly. The response without the lens is indicated in the center of the figure. Although the APD itself has a diameter of only $0.5 \mathrm{~mm}$, the lens collects light over about $1.2 \mathrm{~mm}$

17. Variation in APD response as a function of temperature. The data were taken by monitoring the system for an extended period of time while the temperature went through its normal cycle. The line respresents a least-squares fit.

18. The gain variation of three channels of APD as the global bias voltage supply is varied. The lines are the results of quadratic fits to the data. The error bars represent erros on the means of the distributions. 


\subsection{INTRODUCTION}

During the past year the Superconducting Super Collider Tracking Group has progressed from acquisition of its first avalanche photodiode (APD) to installation of a 96-channel array of the devices. The work was motivated by the desire to leam how to use APDs as the sensitive elements in a fiber tracking detector, moderated by the presence of limited resources and the absence of activity within groups outside the SSC Laboratory on such a project. We chose, therefore, to team up with an ongoing research effort which intended to evaluate both pre-shower and shower-maximum detectors and various means of sensing the light produced. ${ }^{1}$ The pre-shower detector is made of layers of scintillating fibers similar to a fiber tracker. The shower-maximum detector uses optical fibers to transmit the light from scintillating plates to the readout devices. Our contribution has been to develop the APD array for use in this test from concept to operation. Currently, the equipment is installed in Fermilab's MP beamline awaiting delivery of the final 36 APDs and exposure to the beam.

\subsection{GENERAL CHARACTERIZATION OF APD OPERATION}

An excellent review of avalanche photodiode operation and applications is given by Lightstone and McIntyre. ${ }^{2}$ Here we provide a brief outline.

Avalanche photodiodes have been available on the market for many years, but have generally been considered uninteresting devices for high-energy physics because of their high cost and either low gain $(\sim \times 200)$ or low repetition rate (few $\mathrm{kHz}$ ). Without much effort, however, either a much higher repetition rate or a much higher gain can be achieved. At present the cost of APDs ranges from about $\$ 50$ to about $\$ 200$ per device, although this is anticipated to drop for large quantities. They possess excellent quantum efficiency, peaking around $80 \%$ for light in the $800-\mathrm{nm}$ range and dropping to about half that in the blue. (Compare photomultiplier tubes, which are typically about 5-8\% in the blue.) Figure 1 shows the spectral response curves for two different APDs.
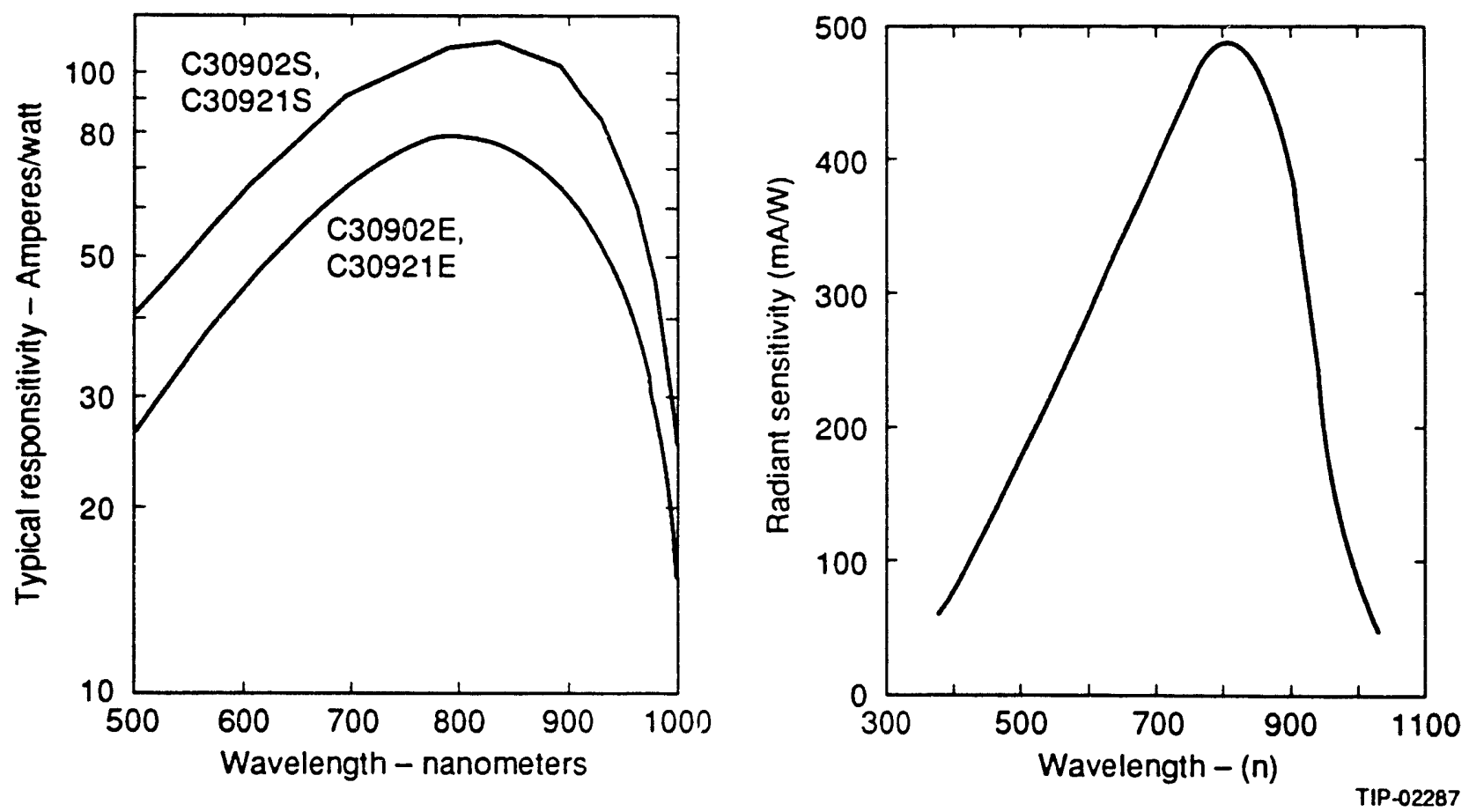

Figure 1. Spectral response curves for two commerclal APDs. 
The gain mechanism in APDs is an electron avalanche occurring in a high-field region of a thin silicon wafer. The high field develops in the depletion layer of the diode when a reverse bias is applied. As the reverse bias voltage is increased, the thickness of the depletion layer increases until it extends all the way through the diode. The external bias at that point is called the depletion voltage. An electron liberated by a photon striking a silicon atom in the $P$ side of the diode will be accelerated toward the $\mathrm{N}$ side by the electric field in the depletion layer. Gain is developed as the electron is accelerated sufficiently to liberate additional electrons in collisions with the silicon lattice. The newly liberated electrons are also accelerated, strike atoms in the lattice, liberate more electrons, and so on.

If the electric field is increased above the breakdown voltage, then there is a significant probability that photons will be generated during the avalanche which will initiate independent avalanches in a different region of the APD, eventually involving the entire diode in the avalanche unless some external device limits it. This type of operation is referred to as Geiger mode. Below the breakdown voltage, the diode current increases linearly with increasing incident light, making it useful for measurements of light intensities as required for calorimetry. Figure 2 shows an oscilloscope trace from an APD operated in Geiger mode. The test circuit used to obtain this trace is given in Figure 3. The APD used in these tests is an EG\&G 30902S. ${ }^{3}$ In linear mode the gain is limited to a factor of a few hundred, so that the signal resulting from a single photoelectron is only a few hundred electrons. Observing such a tiny signal requires high-z.din, low-noise electronics. The device manufacturer indicates that the total charge is collected in under $1 \mathrm{~ns}$ in this mode, but this is nontrivial to demonstrate because any observable signal is dominated by the associated electronics. Our experience with linear mode signals wili be described more fully in the following sections.

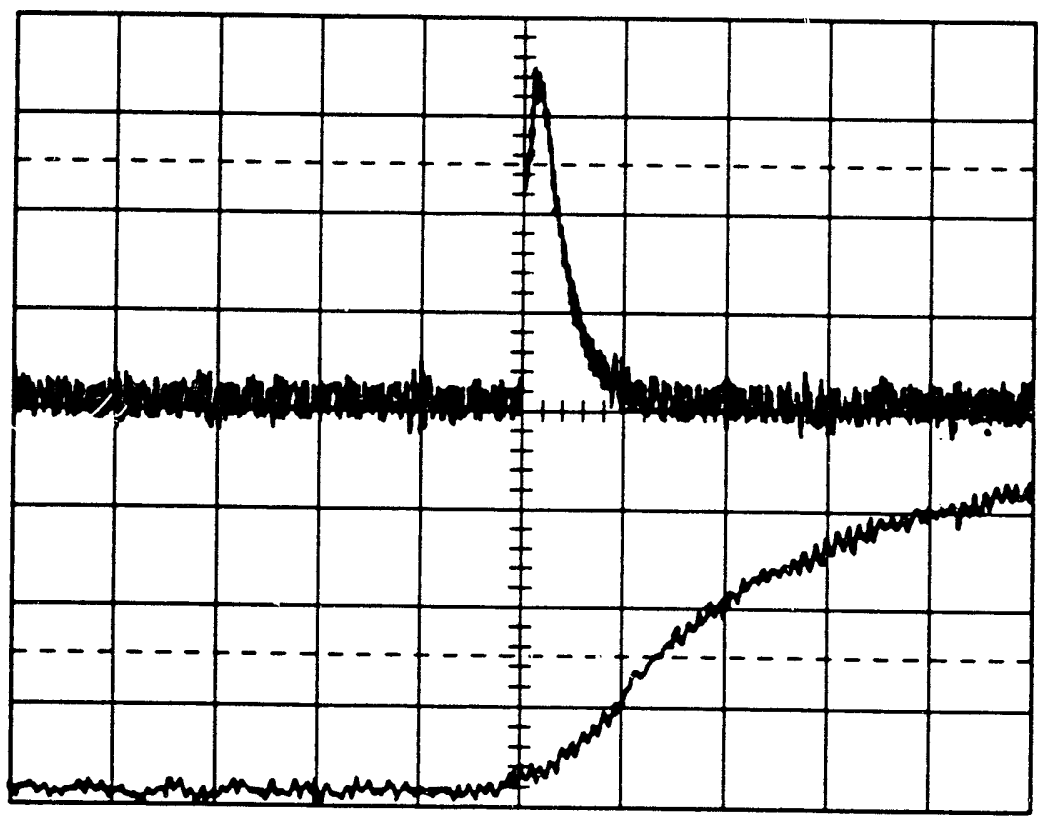

$5 \mu s / d i v$ $50 \mathrm{mV} / \mathrm{div}$

$100 \mathrm{~ns} / \mathrm{div}$

$50 \mathrm{mV} / \mathrm{div}$

TIP-02288

Figure 2. Oscllloscope trace showing the signal from an avalanche in an APD operated In Geiger mode. The same trace is shown at two different time scales. For thls response the APD was discharging into $240 \mathrm{~K} \Omega$. 


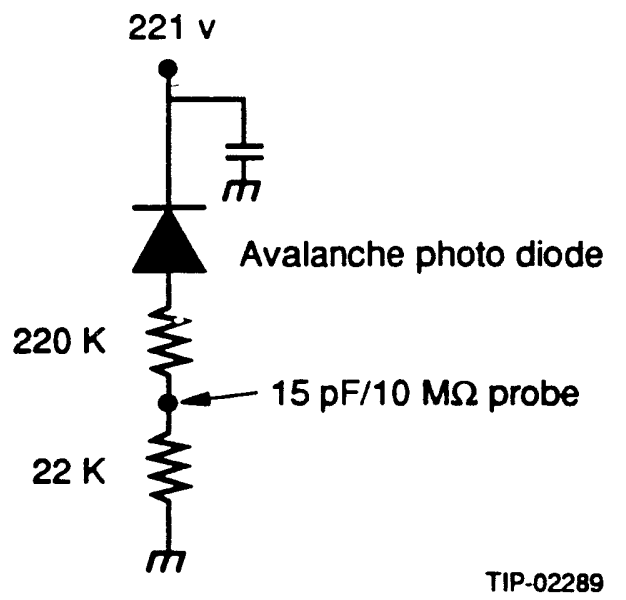

Figure 3. Test circult used for observing Gelger niode APD signals.

\subsection{SENSITIVE ELEMENT REQUIREMENTS FOR PRE-SHOWER/SHOWER- MAXIMUM DETECTORS}

Pre-shower detectors are placed as the first layer in a calorimeter system and are intended to observe the incoming charged particle, if any, as well as the first stages of the electromagnetic shower. They are effectively a cross between a tracker and a calorimeter in that ideally they provide a precise measurement of the positions of charged particles as they enter the calorimeter without sacrificing undetected energy loss. Given the data collected by pre-shower and shower-maximum detectors, one can also make powerful cuts on particle identity: e/ $\pi, \pi \% \gamma ;$ e/ $\gamma$. The spatial separation between identifiable particles can be as small as the segmentation of the detector, or down to $\sim 500 \mu \mathrm{m}$.

In a pre-shower or shower-maximum detector built of scintillating fiber, then, the requirements on the sensitive elements are: 1 ) small size in order to mate naturally with the large number of small detector elements; 2) linear response to deposited energy in order to serve as part of a calorimeter, 3) sensitivity to typical minimum-ionizing particle energy deposits; 4) sufficient dynamic range to avoid saturation at shower maximum; and 5) speed consistent with the event rates expected in the experiment. It appears that certain ADPs may possess the necessary requirements for such detectors. 


\subsection{APD LINEAR CIRCUIT DEVELOPMENT}

For detector development tests in a low-intensity test beam, it is not necessary to optimize the shaping time or repetition rate characteristics of the APD electronics. Therefore we chose to use a conventional charge-sensitive amplifier ${ }^{4}$ utilizing low-noise FETs biased for optimum noise characteristics. Initial testing of this concept was performed using devices built for the DO experiment at Fermilab. ${ }^{5}$ The schematic is shown in Figure 4.

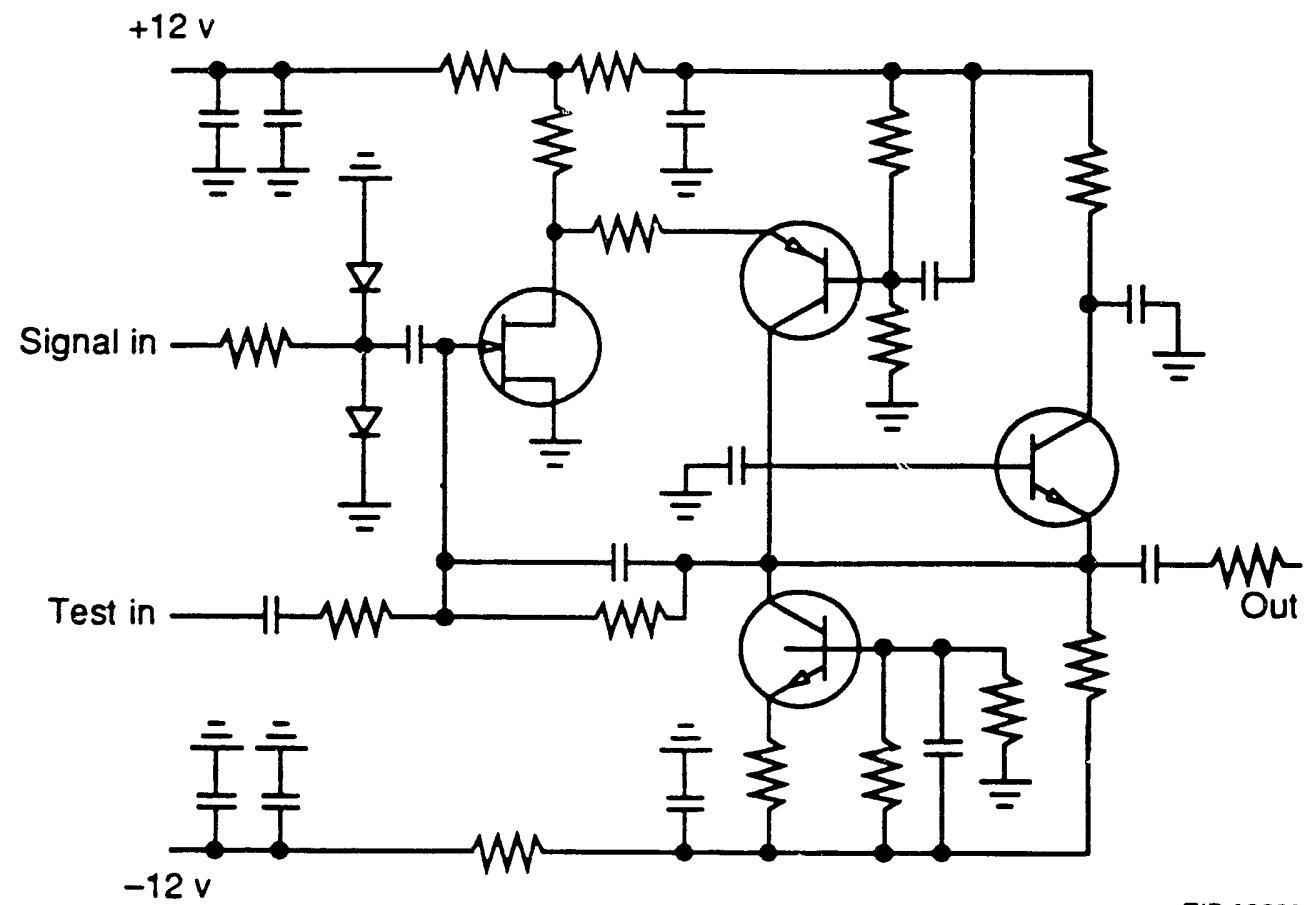

Figure 4. The DO charge-sensitive preamp circuit used in initial linear mode tests of APDs.

The tests of the APD and amplifier used a green LED $(565 \mathrm{~nm})$ to excite the photodetector, and a photomultiplier tube (PMT) preamp (LeCroy6 612; ad a current-integrating analog-to-digital converter (LeCroy 2249A) to record the in-time output of the system. The LED was driven by short $(\sim 10 \mathrm{~ns})$ pulses of $\sim 175 \vee$ coupled to the LED through a capacitor. Some fraction of the light output of the LED was transmitted by a 1-mm-diameter plastic optical fiber to either the ADP (for testing) or to a PMT (for calibration of the light intensity). Because of their simplicity and low cost, AMP OPTIMATE series ${ }^{7}$ connectors were used for the optical couplings in the system. It is also simple to fabricate mating optical sockets for these connectors (see below). The overall setup is shown in Figure 5. 


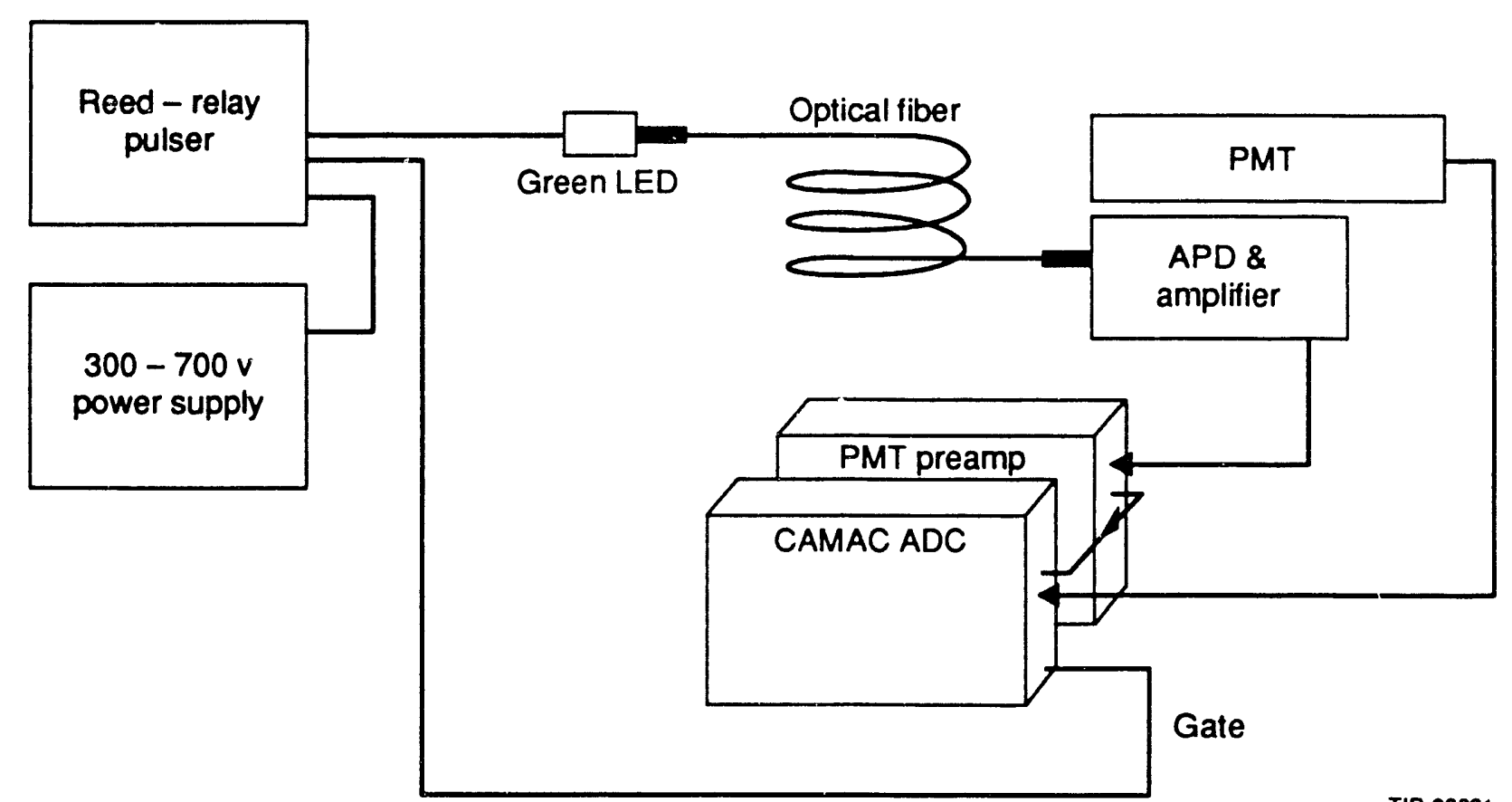

TIP-02291

Figure 5. Block dlagram of experimental arrangement for Initlal APD Siudies.

Figure 6 shows two superposed histograms of the current integrated out of the electronics during a gate triggered by the light flasher. The lower histogram is the system response with no light flash; the upper histogram shows the response to a light flash determined later to be about 60 photons. This was the initial test of the system to determine whether it was at all sensitive to the test pulses of light.

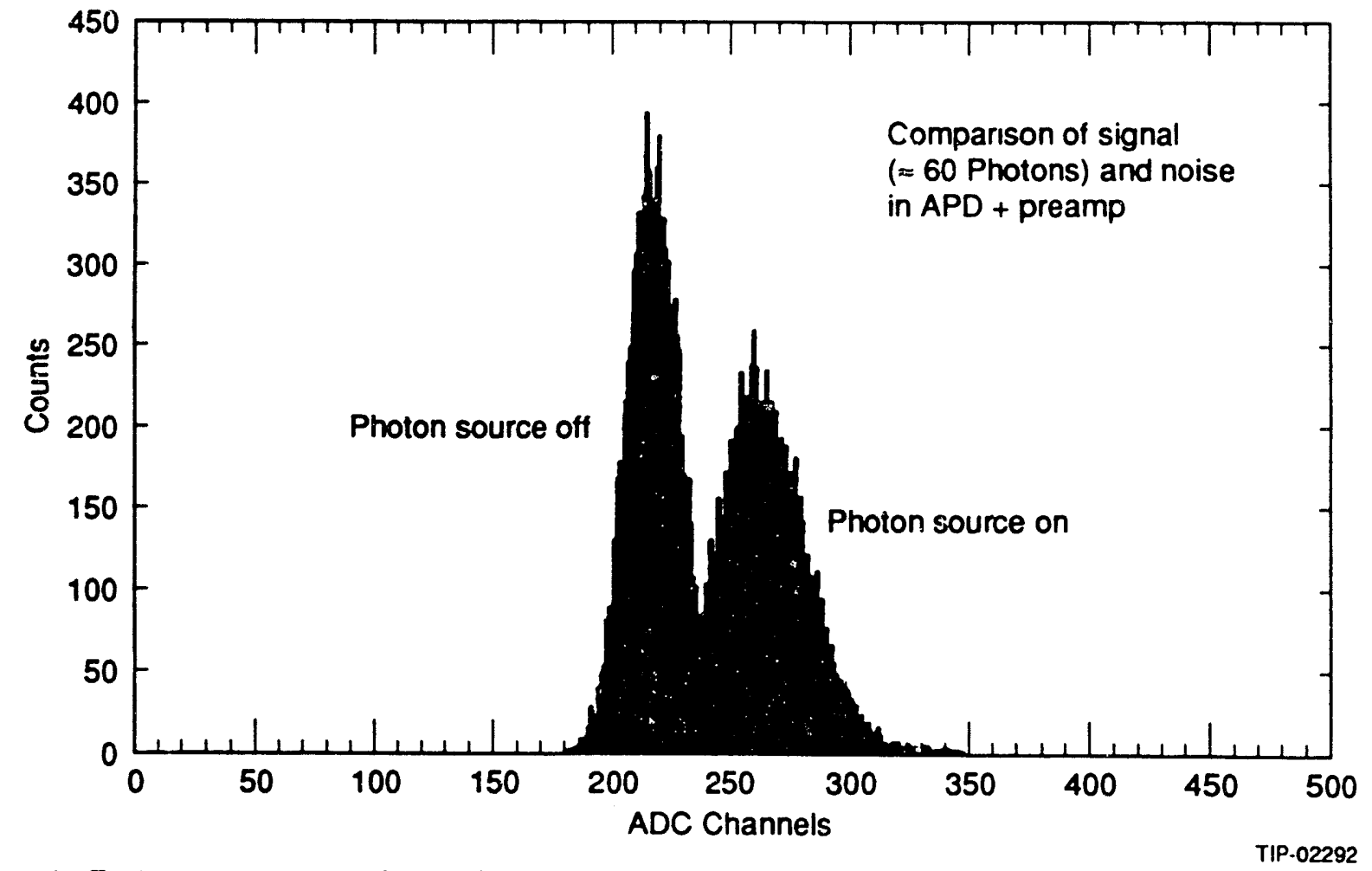

Figure 6. Early test resulte of an APD operating in linear mode and exposed to about 60 green $(565 \mathrm{~nm})$ photons. The electronic noise apparent in thls figure was later reduced. 
Further testing established the linearity of response of the system to differing light levels. To do this, the LED flasher supply voltage was varied over several hundred volts and the avera ${ }_{6}$ e APD/amplifier response was determined at each one. Then, data was taken at the same set of LED flasher voltages with the light input and signal output connected to a phototube instead of to the APD. In this way it was possible to estimate th : number of photons incident on the surface of the APD (or PMT) at each LED flasher voltage. The PMT used for these tests was a Philips XP2020 20 with a quantum efficiency of $\sim 6 \%$ at $565 \mathrm{~nm}$. The APD under test has a circular sensitive area $0.5 \mathrm{~mm}$ in diameter, so that the APD sees at most $25 \%$ of the light emitted by the 1-mm-diameter fiber. When the fiber is attached to the PMT, all of the light strikes the PMT surface. (We determined that any reflection losses at the fiber-APD or fiber-PMT window interfaces must be small since no measurable change in the response of either detector was observed when optical grease was used at the interface. A fine polish was maintained on the end of the fiber, and all tests reported in this paper were made with dry optical contacts.) Thus, if $N$ photoelectrons were produced in the phototube, there were N/0.06) photons at the PMT surface and at most $(0.25 \cdot N / 0.06)$ photons incident on the APD surface. Figure 7 shows the APD system response to $565 \mathrm{~nm}$ light of intensity ranging from 0 photons to 1000 photons per pulse. In order to span this dynamic range of intensity we used two different LEDs having different coupling efficiencies to the optical fiber. The light intensity from each LED was calibrated as described above.

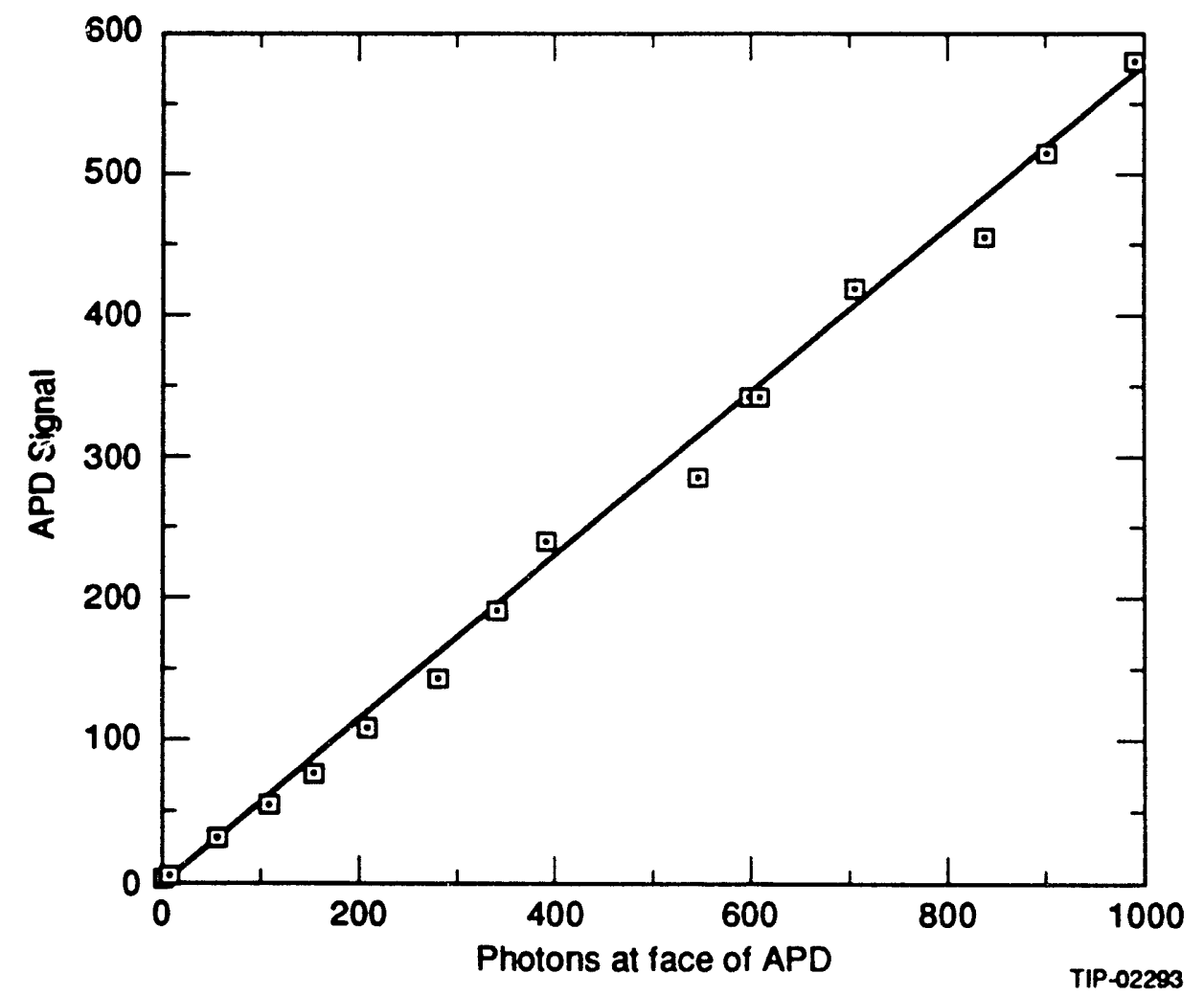

Figure 7. Response of an APD to varying amounts of incident light. The scatter of the data above and below the straight line was reduced by stablizing the temperature of the APD. 
It is apparent from Figure 7 that there is a linear relationship between the number of photons striking the APD and the integrated charge out of the APD electronics. The data are scattered around the best line fit, however, more than statistical errors alone would suggest. It is known that the breakdown voltage-and, therefore, the gain at constant bias voltage-varies with APD junction temperature. Typical specs give a voltage coefficient for constant gain of about $0.5 \mathrm{~V} / \mathrm{C}^{\circ}$. When the linearity tests were repeated with the APD can inserted in a brass bar cooled by tap water $\left(\sim 20^{\circ} \mathrm{C}\right)$, the gain was observed to remain constant in time Figure 8 shows the response as a function of time before thermal control was applied. From this work it became obvious that some form of temperature control or compensation would be required in order to make practical use of the APDs in the final system.

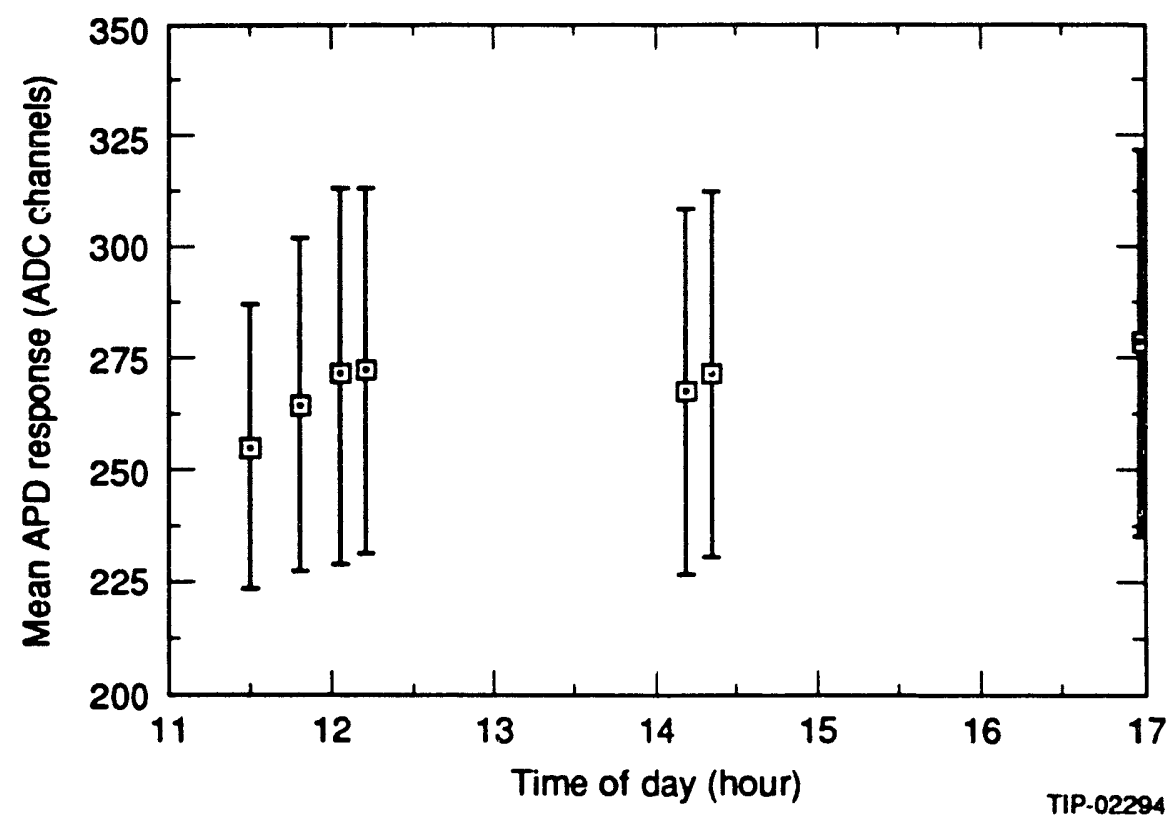

Figure 8. Varlation of APD response with time of day. Thermal stablilzation of the APD eliminated this varlation. Note that error bars indicate the statistical spread in the signais. The error on the mean is of order one ADC count.

\subsection{APD ARRAY SYSTEM DESCRIPTION}

\subsection{Electronic}

A printed circuit board was fabricated implementing 16 copies of a charge-sensitive amplifier similar to the one studied initially. Provided as the final stage for each channel on the card is a differential ECL line driver (10216) used in the linear range. Bias voltage for each APD is adjustable over a range determined by a zener diode on the board, so that only one supply is riecessary to bias all 16 channels even though they each may require different voltages. Temperature monitoring of the APDs is achieved by attaching an RTD to the metal assembly supporting the 16 devices. The RTD resistance, directly proportional to the 
temperature, is converted to a voltage by a $1-\mathrm{mA}$ current source. The voltage is amplified for noise immunity and sent out to a monitor panel along with a sample of the low-voltage power present on each card. The voltage monitors are necessary for practical system operation since safe engineering practices require fusing the power supply lines on each circuit board. The schematic of this circuit is shown in Figures $9 \mathrm{a}$ and $9 \mathrm{~b}$. Figure 10 is a photograph of one of the cards with the shielding removed.

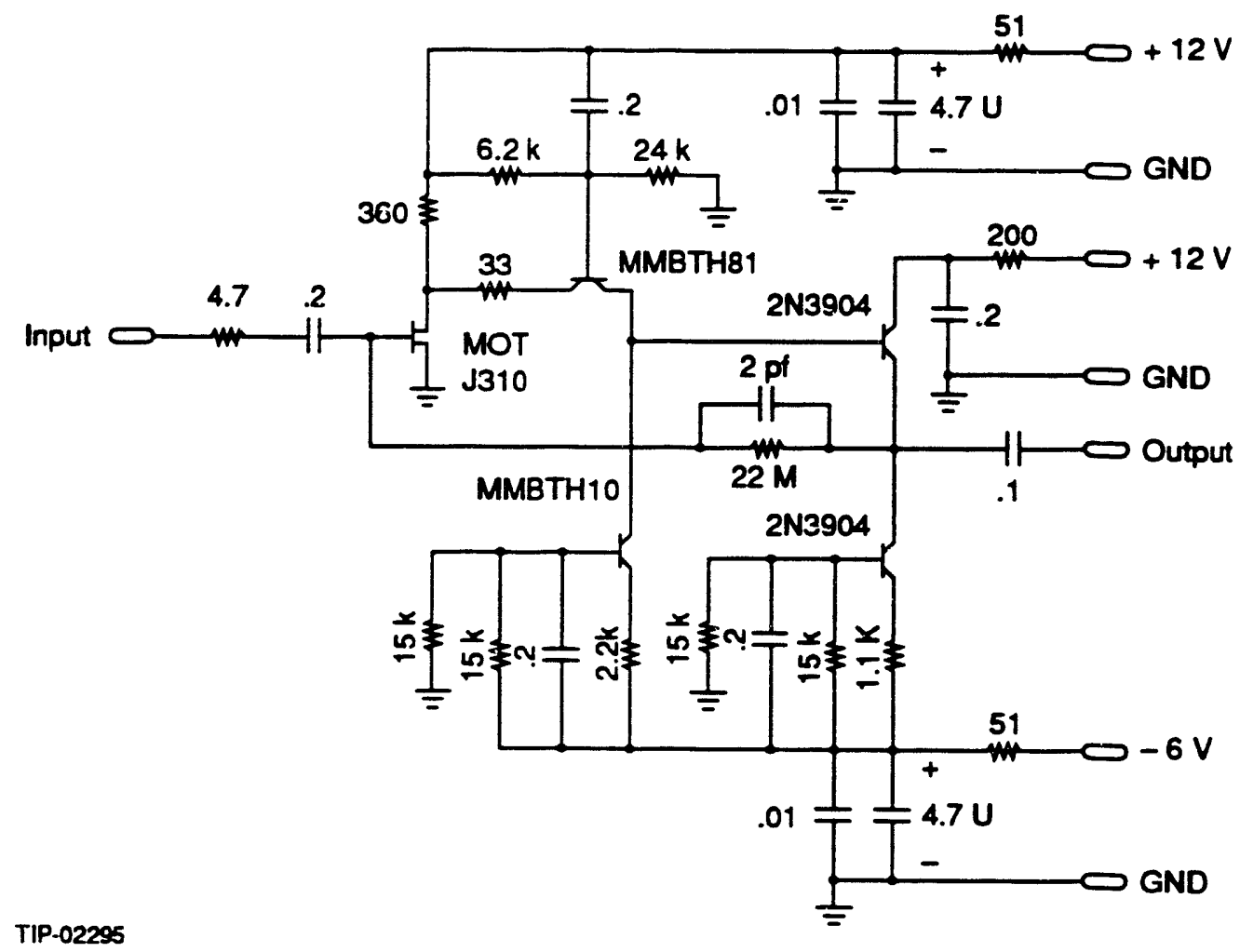

Figure 9a. Charge-sensitive amplifier circuit implemented in the 96-channel APD system. 


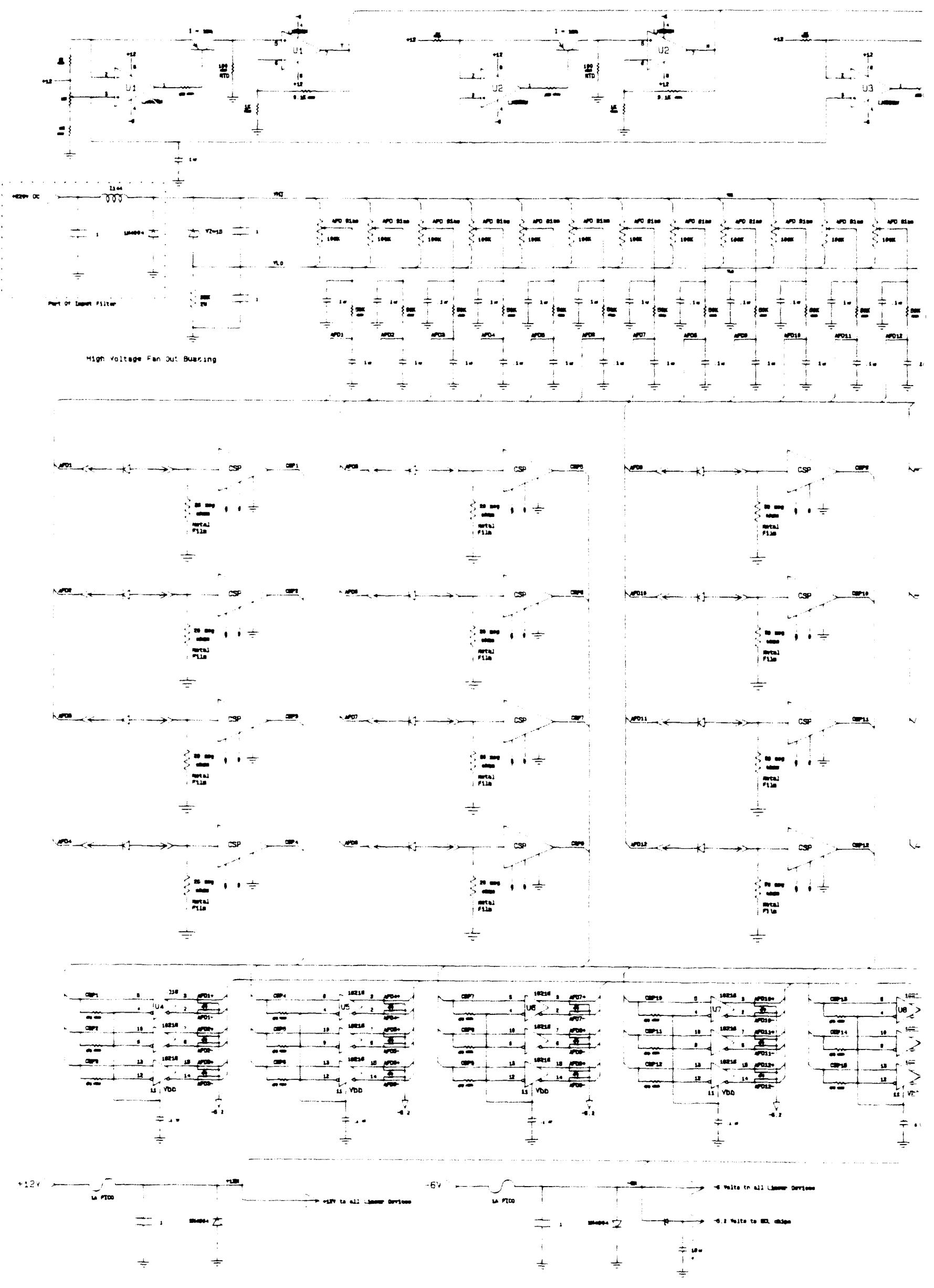



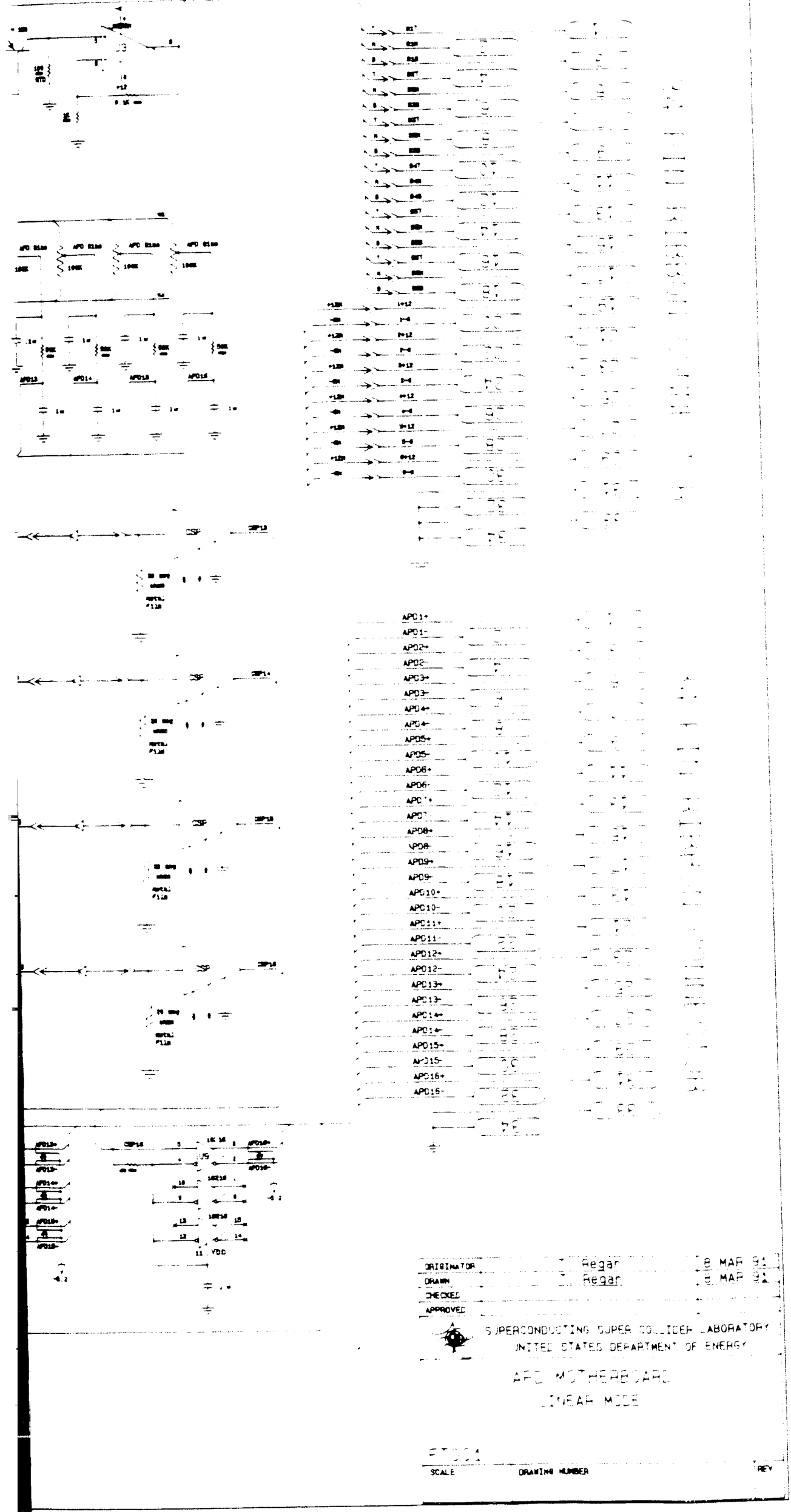

Fig. 9b. Schematic diagram of a 16-channel APD/Amplifler/Driver circult board. The three blocks at the top are the temperature monitoring circults. 


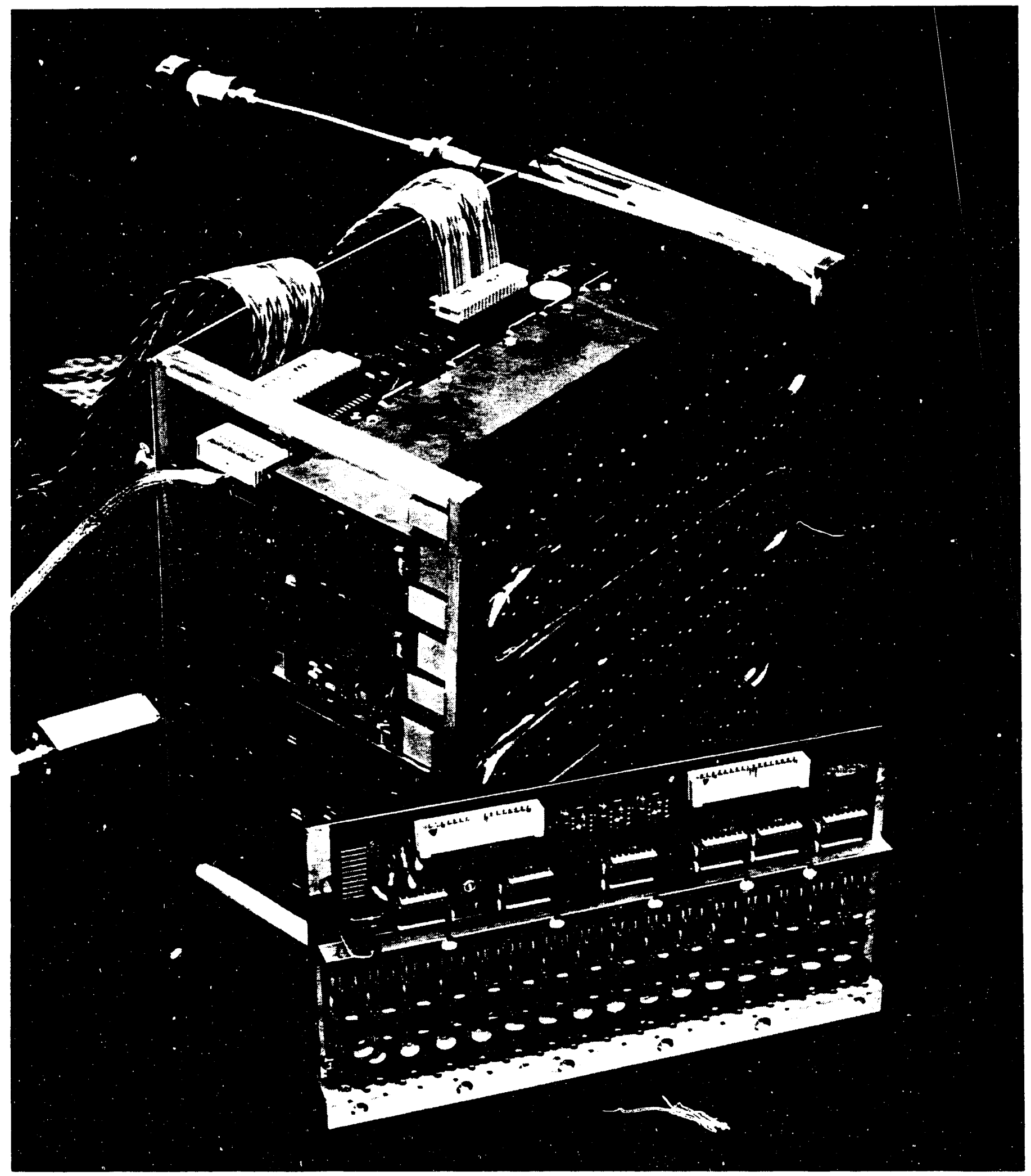

Figure 10. Photograph of the 96-channel array composed of six cards of 16 channels each. One card is shown with the RF shield removed. The APDs are imbedded in the bar at the front of each card. Optical connectors, one with flber attached, are shown Inserted In the topmost card. Thermal stability ls provided by circulating water through the plpe attached to the front of the crate. 
The resulting differential signals are transmitted over twisted-pair ribbon cables (about $30 \mathrm{~m}$ long) to the digitizing electronics. Truly isolated differential inputs are required at the receiving end to maintain acceptable noise levels in the test beam area and to ease the elimination of ground loops. This termination is provided by installing pulse transformers at the front panel of the LeCroy 1885F ADC, used in the data acquisition system.

\subsection{Mechanical}

Given that the system had to mate with an existing detector design, there were constraints imposed on overall size, net heat generation, RF noise production, layout and method of the optical connections, and means of communicating signals and utilities through the walls of the light-tight detector enclosure. Figure 11 is a working drawing of the entire detector and enclosure with APD system conceptual design shown in place.

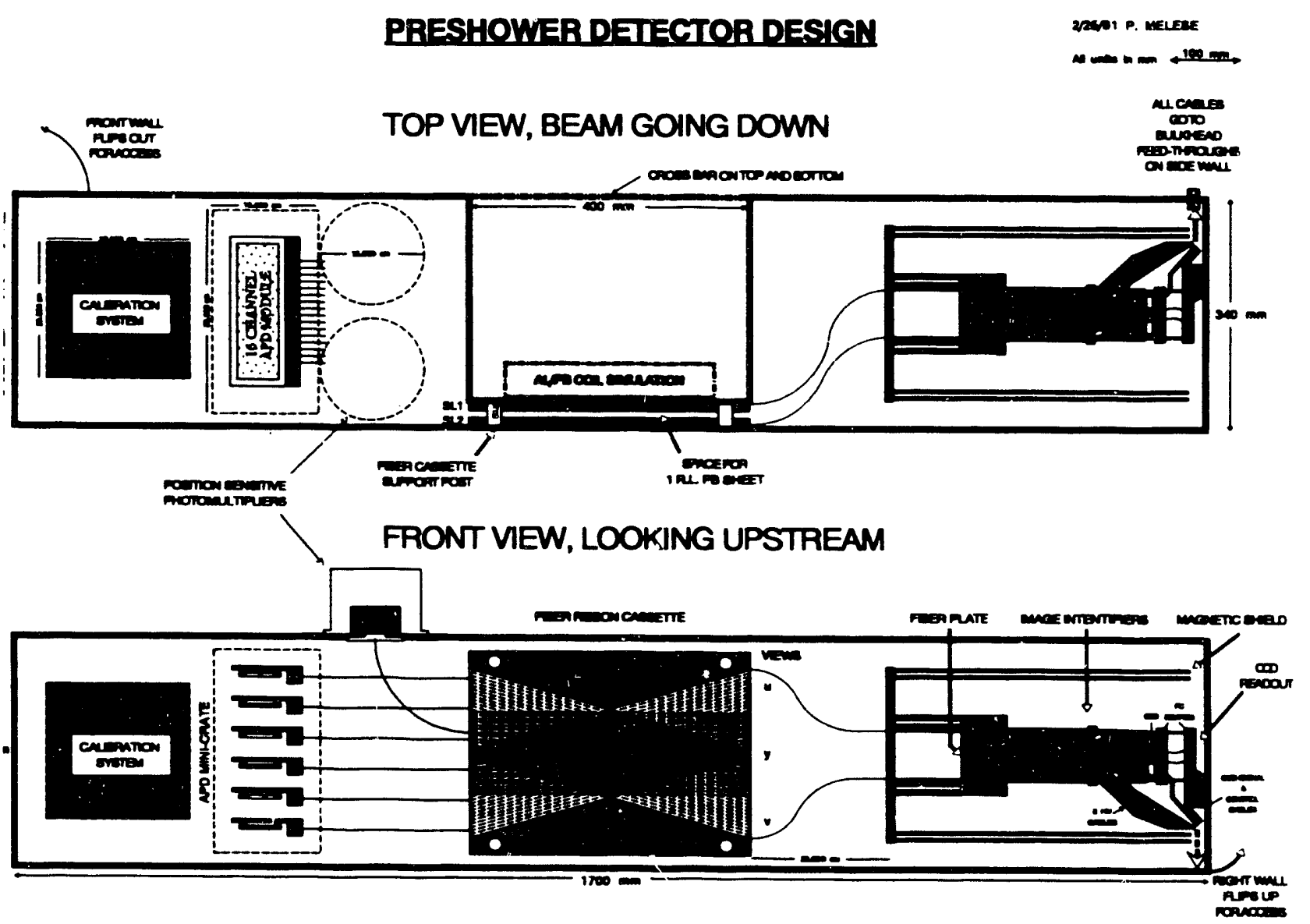

Figure 11. The Pre-shower Detestor. Scintlllating fiber rlbbons are in the middle and are read out by various test devices on either end. The entire assembly is enclosed in a light-tlght box. 
Both to control the temperature of the APDs and to remove any heat generated by the electronics, chilled water was cycled through a copper pipe brazed to brass bars, which in tum were clamped in thermal contact with the metalwork surrounding the APDs. Figure 12 demonstrates this design. The metalwork was fabricated so as to accept the AMP optical connectors attached to the scintillating fibers and to align the fibers with the windows on the APDs. Also shown in this figure is the massive RF shielding incorporated on each circuit board. The circuits are inherently quiet, but quite sensitive to ambient RF noise because of the low APD signal levels.

As yet undefined trame

Incoming beam side

aka: front

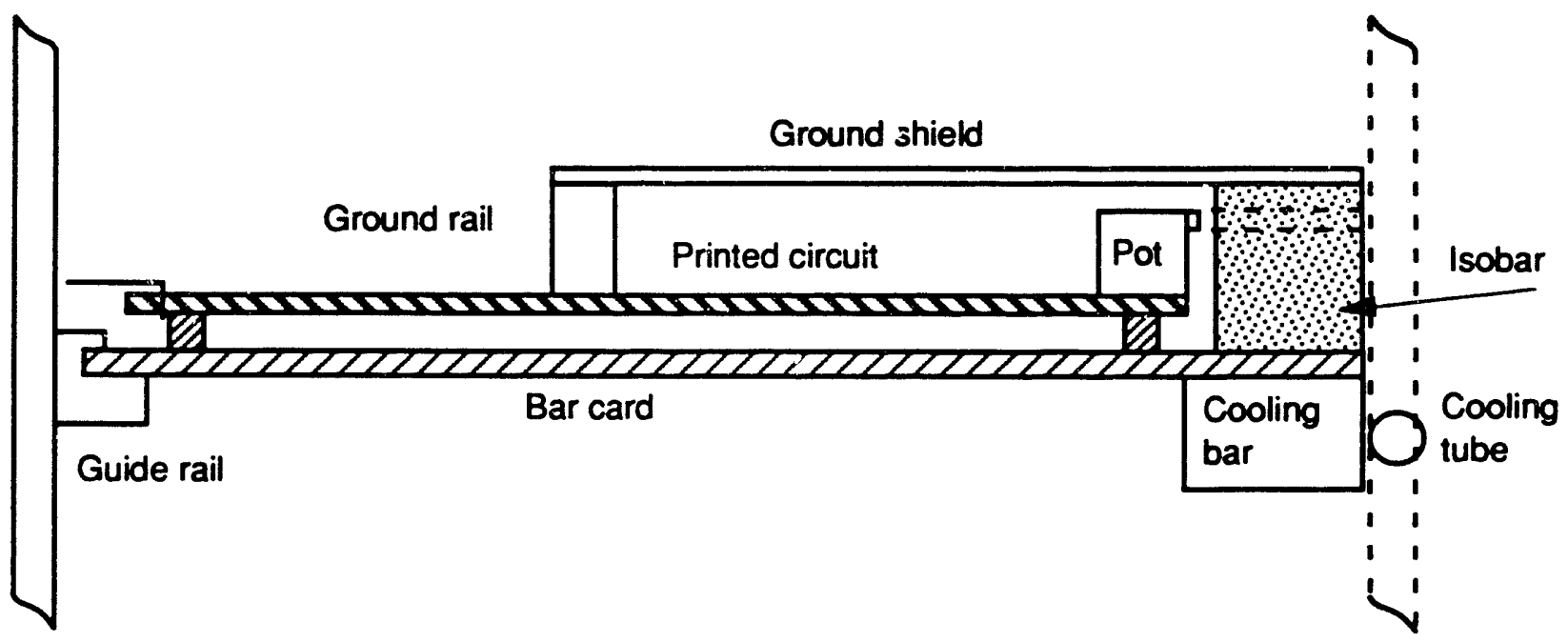

TIP-02296

Figure 12. Detall of the assembly of the electronic circult board to the cooling system and crate. The avalanche photodiodes are mounted in the "Isobar."

Feed-through connectors for chilled water, signals, power, and status monitoring were mounted on a special light-tight printed circuit board. Specifications for this panel were sent to the institution fabricating the detector enclosure so that a mating hole could be designed into it. This board is shown in Figure 13. 


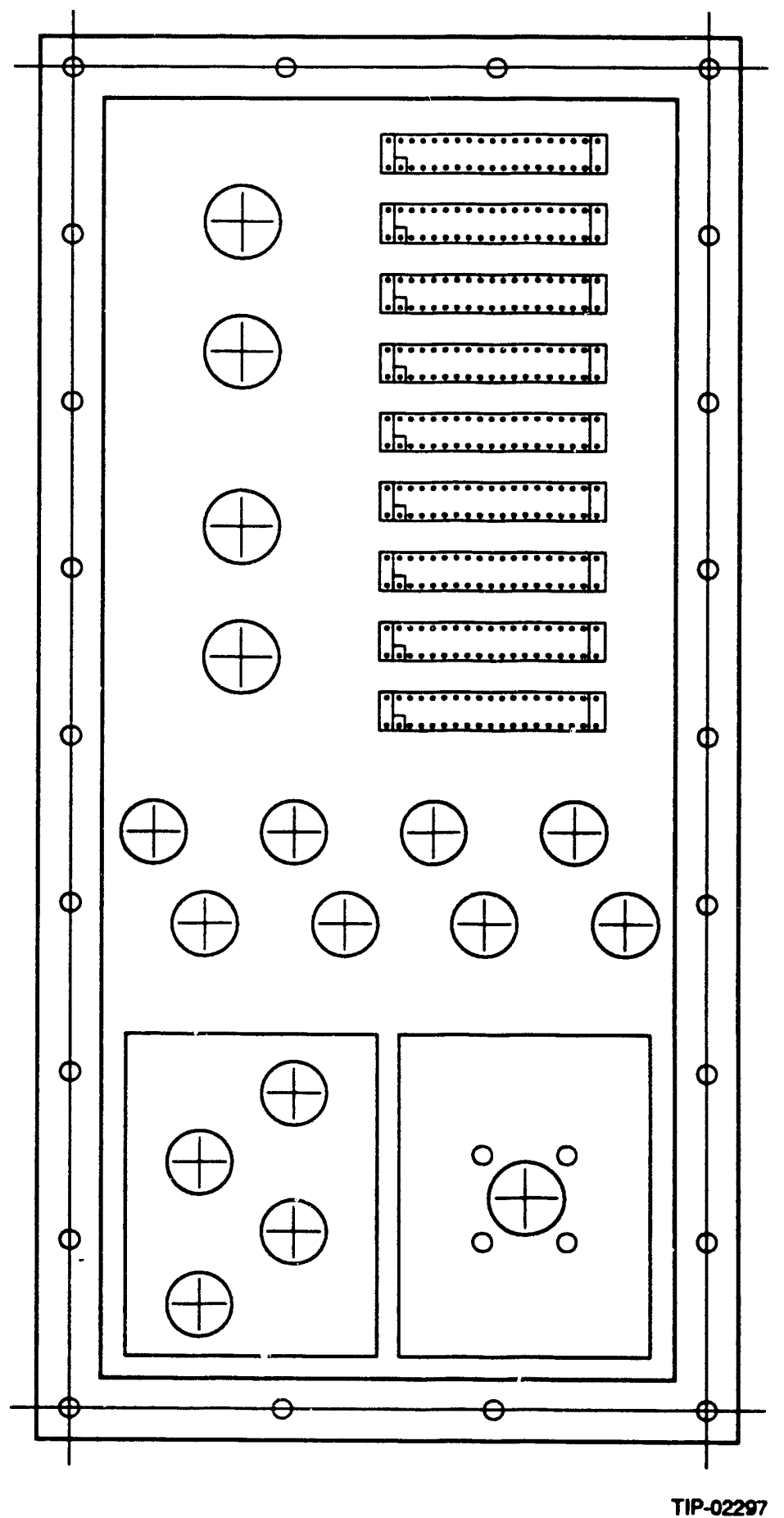

Figure 13. The Fend-Through Panel. Constructed of copper-clad black substrate, thls panel provides light-tight bulkheads for elght ribbon cables, four gas or water lines, eight BNC cables, four SHV cables, and a multiconductor low-voltage connector. 
Six assemblies of circuit boards and APD mounting bars were fabricated. Spare circuit boards were prepared as well. The cards slide into slots in a custom-designed crate. One side of the crate is made up of heavy brass bars soldered to the chilled water pipe described above. The APD bars are clamped tightly to the chiller bars by commercial clamping fixtures to provide a secure thermal connection. Power, signal, and monitor cables are attached to each card by individual cables from the feedthrough panel. A photograph of the entire assembly is shown in Figure 10.

\subsection{Optical}

The same optical connectors used in the early bench tests of the APD were chosen for use in the full system. The AMP Optimate connector, shown with the mating hole, fiber, and APD in Figure 14, provides a convenient solution for a system of this size ( 96 channels total). The corinectors are designed for $1-\mathrm{mm}$ fibers having a large-diameter plastic sleeve around them, however. In order to attach them to fibers without a sleeve, the special plastic taper shown in the figure was developed.
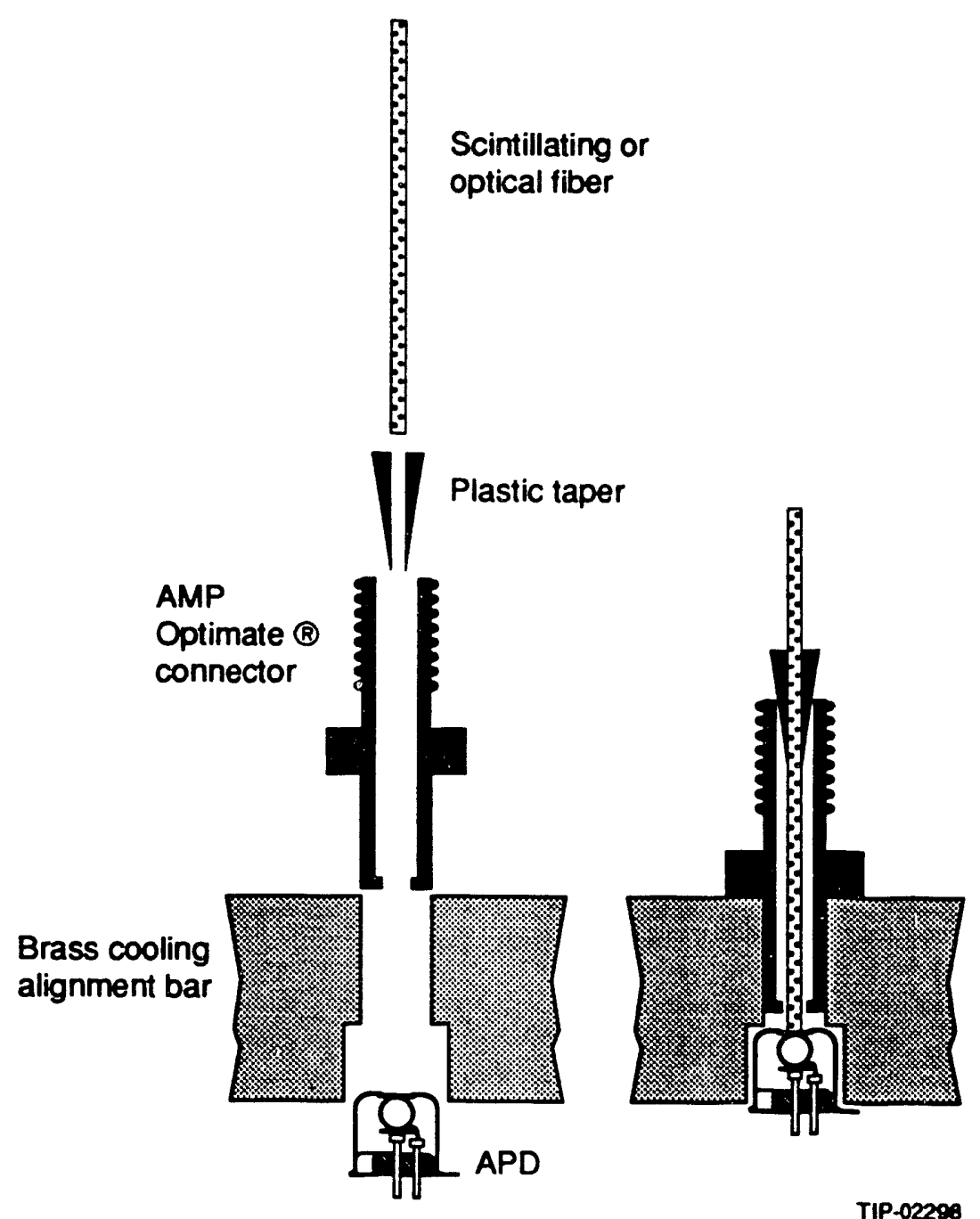

TIP-02290

Figure 14. Optlcal Alignment Procedure. The APD is mounted in a thermally stablized brass bar. 
The standard APD assembly from EG\&G is a metal can with a window in the center of the flat surface over the sensitive pri junction. Special devices were designed and fabricated by EG\&G for this system to improve the optical coupling efficiency from the $1-\mathrm{mm}$-diameter scintillating fibers to the 0.5 -mm-diameter diode. Figure 15 shows the solution. The photodiode and optical winciow are placed within $50 \mu \mathrm{m}$ of the axis of the case to allow the outer diameter of the case to be used as an alignment reference. A spherical glass bead is mounted between the diode wafer and the window. This focuses light from the periphery of the fiber onto the APD surface, providing an effective sensitive area of diameter $1.2 \mathrm{~mm}$. A map of the APD's response across the surface of the window is shown in Figure $16,9^{9}$ which is a composite of the results of two scans: one with the ball lens in place and one without the lens. It is clear from this figure that the lens botl widens the effective aperture of the APD and provides a more nearly uniform response over the active area.

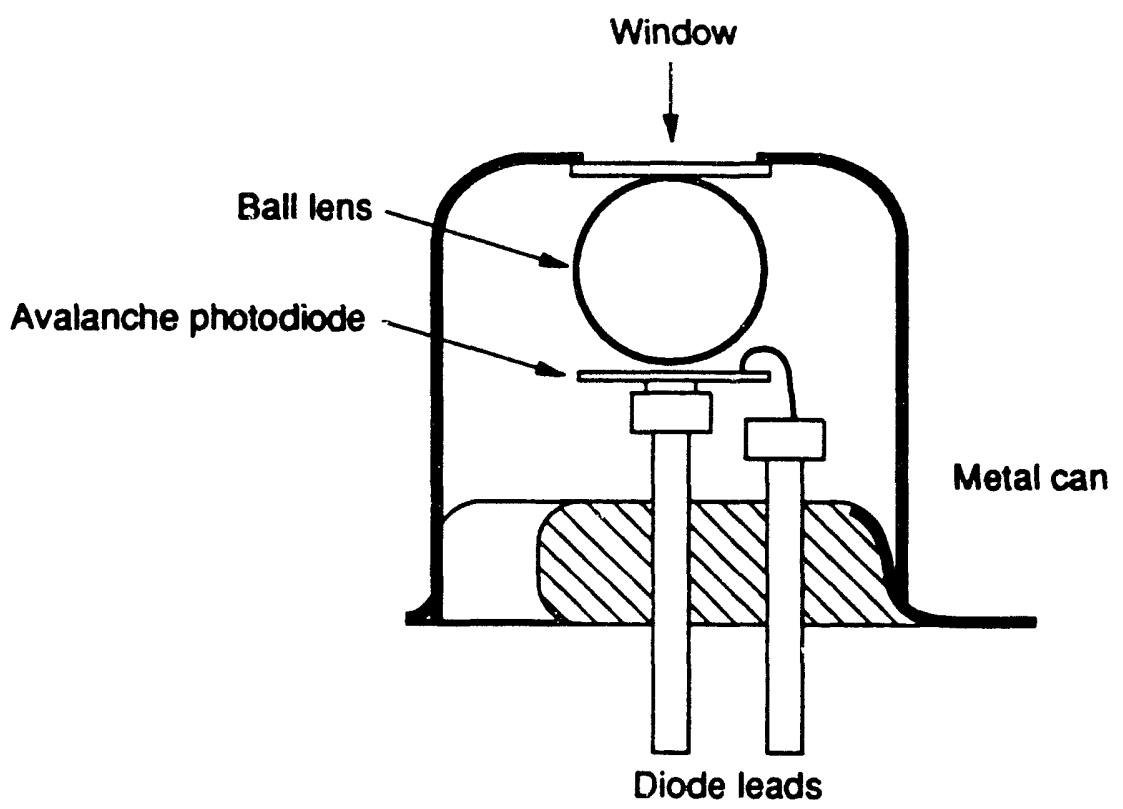

TIP-02299

Figure 15. Cross section of the APD deveioped by EG\&G Optoelectrori'ts for use with 1-mmdiameter fibers. The APD ltself is only $0.5 \mathrm{~mm}$ wide. 


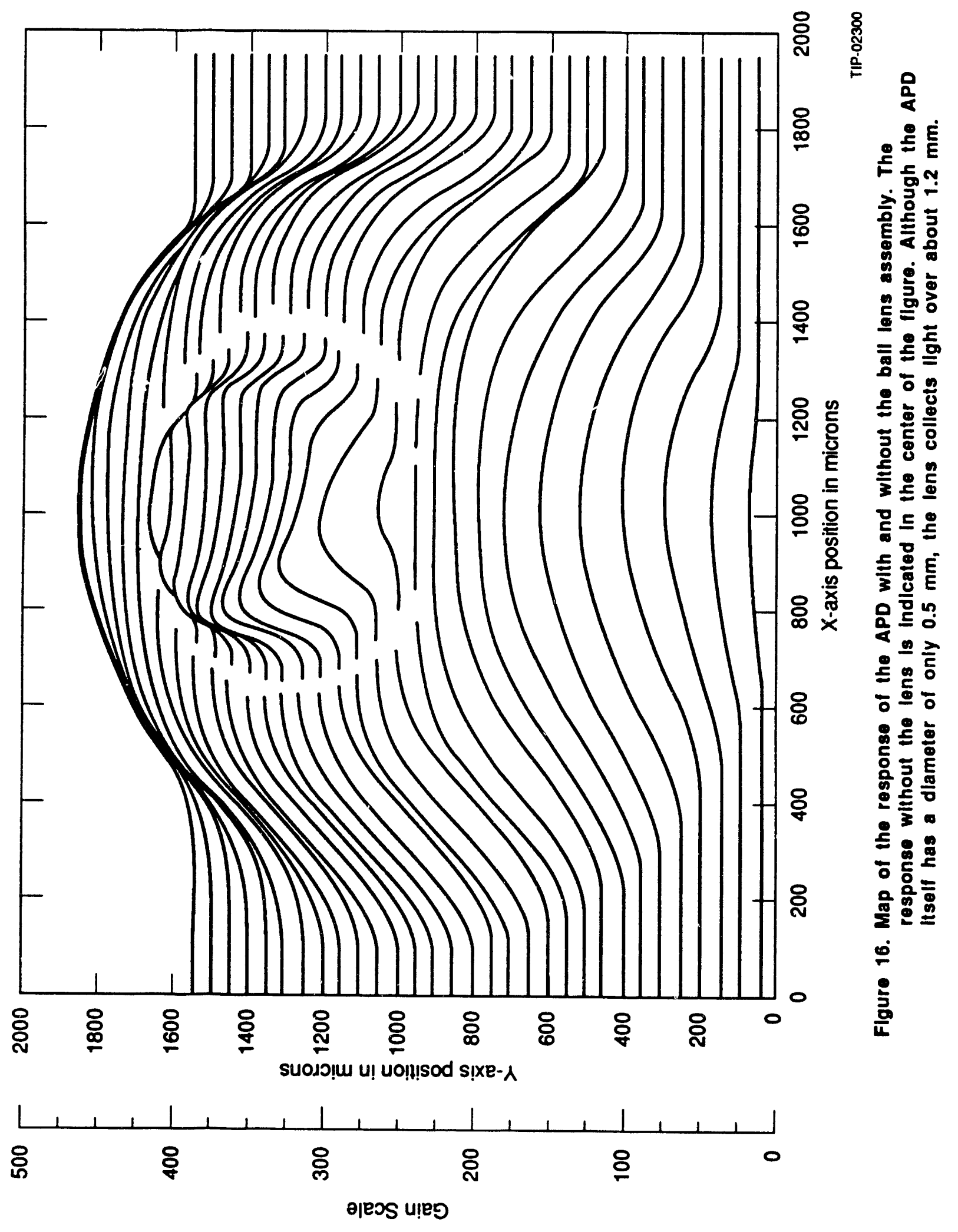


The fiber ends were prepared by cleaving them with a cutter designed to maintain a flat, perpendicular surface. They were then polished with the aid of a holding fixture, using a soft buffing surface. Finished fibers were inserted through the custom-tapered plugs and the plastic optical connectors. The tapers were driven home with the fiber-end protruding 250-500 $\mu \mathrm{m}$ from the end of the connector. This protrusion was chosen to ensure that the contact between the end of the fiber and the APD assembly window provided the mechanical stop, ensuring no gap in the light path. This system is very convenient to use because the optical connections are simple to make and are automatically light-tight.

\subsection{System Test Results}

As of this writing, the system is installed in the MP hall at Fermilab. It will be attached to the pre-shower detector and placed in the beam in the near future. Meanwhile, a number of system tests have been performed in preparation for actual data-taking.

It is necessary to set the gain of each channel in the entire assembly to an appropriate level. Although channel-to-channel gain variations could be accommodated at the time of off-line analysis, it is preferable to set all charmels to the same value. In the present arrangement this is accomplished by adjusting the bias voltage of each APD so that the same signal is observed from all channels when each is excited by the same amount of light. The level of gain chosen for the pre-shower counter is such that the signal from a minimum-ionizing particle (no shower) can just be separated ( $1 \sigma)$ from the baseline noise. In this way we should be sensitive to the incoming electron track as we measure the full shower energy deposited in each fiber. When the APD array is used with the shower-maximum detector, this gain will be reduced by about an order of magnitude in order to accommodate the larger signals without saturating the ADCs.

This channel balancing has been accomplished with one complication. The thermal control system as arranged while the crate is outside the detector box cycles approximately $\pm 0.4 \mathrm{C}^{\circ}$ with a period of about 10 minutes. Over this $0.8 \mathrm{C}^{\circ}$ temperature range the gain of the APDs, at the setting required for detecting minimum ionizing paricles, varies about 10\%. Figure 17 shows the measured response of one channel as a function of APD temperature. As seen in the figure, the variation is quite linear over this range so that as the temperature of the devices is recorded along with the output signal level, this variation can be removed during analysis.

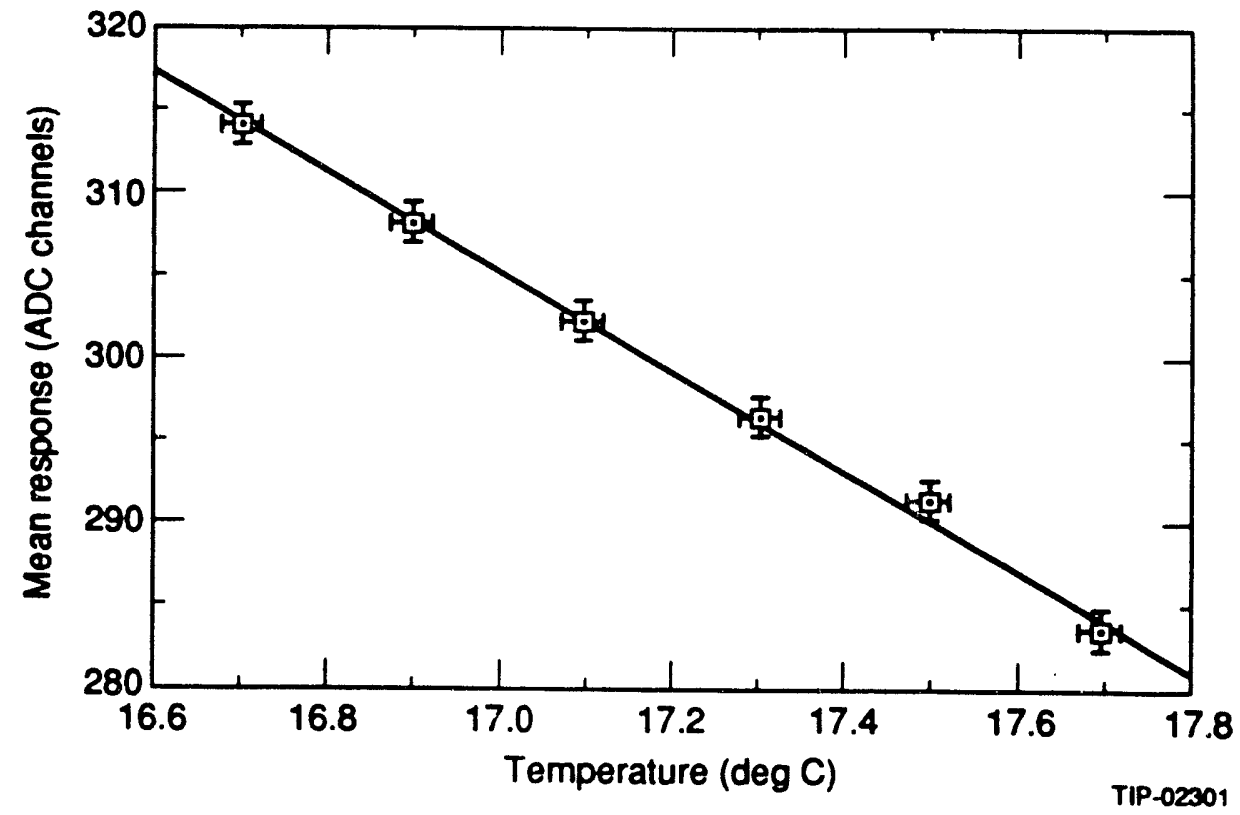

Figure 17. Variation in APD response as a function of temperature. The data were taken by monitoring the system for an extended period of time while the temperature went through its normal cycle. The line represents a least-squares flt. 
When the APD system is moved from the pre-shower detector to the shower-maximum detector, the gains of all channels must be reduced uniformly. That this can be done without laboriously retuning each of the 96 channels is demonstrated by Figure 18, which shows the responses of a group of channels to the same light flash at three different global bias voitage settings. It appears that the gains of all of these channels track one another to within 5\% over a change in average gain of at least a factor of two. Additional tests are needed to determine whether larger uniform gain variations can be achieved.

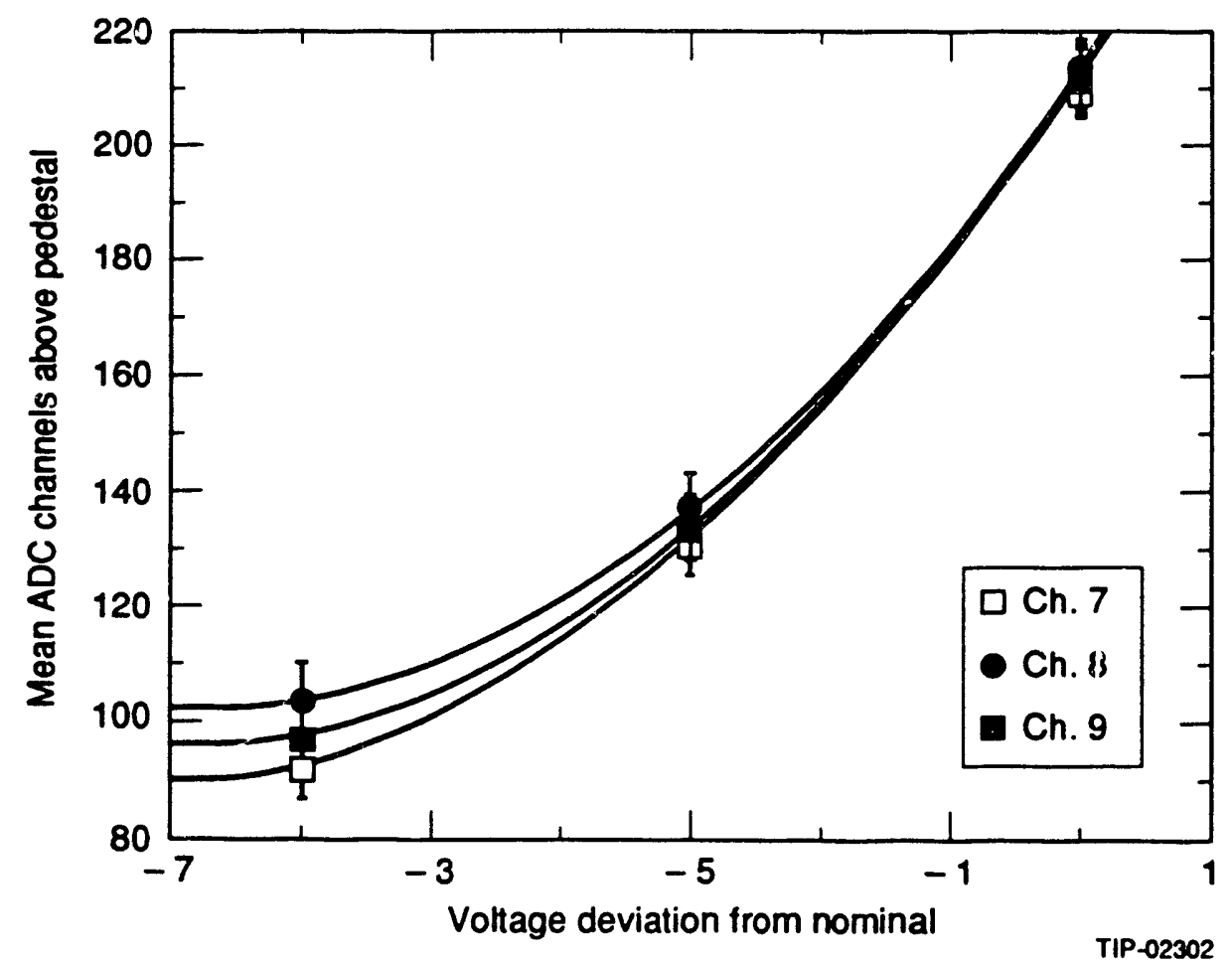

Figure 18. The gain varlation of three channels of APD as the global blas voltage supply is varied. The lines are the results of quadratic fits to the data. The error bars represent errors on the means of the distributions.

\subsection{SUMMARY}

We have designed, built, and begun operation of a 96-channel array of avalanche photodiodes to detect the light produced in the scintillators of a pre-shower detector and a shower-maximum detector. In the process we have observed the relevant characteristics of APDs themselves and have developed practical ways to use them. Appropriate electronics, optical linkages, and environmental controls are necessary for such a system, but none imposes a serious obstacle. We anticipate that data collection in an electron beam will begin shortly and proceed smoothly, and that we will soon be able to report on the results from those tests. 


\section{ACKNOWLEDGEMENTS}

The authors are deeply indebted to numerous researchers and diligent workers within a variety of organizations who have collaborated or provided advice and assistance during the course of this project. In particular, we wish to thank M. Esaili, G. Hale, and C. Mangene of the SSC Detector Physics Lab for their skill, patience, and diligence in developing and constructing the systems used in the tests described. For the pre-shower counter and arrangements for its use in these tests, we are grateful to researchers at Yale University and Rockefeller University. The development of the APD; by EG\&G has been guided by Northeastem University, which provided the devices for this test. M. Hulbert of Northeastem, under the direction of $\mathrm{I}$. Leedom and S. Reucroft, has taken the major responsibility for implementing the APD system in the beamline and for performing most of the system tests and calibration. 


\section{REFERENCES}

1. L. price et al., Fermilab T841 Proposal, 1990.

2. A. W. Lightstone and R. J. McIntyre, Proceedings of the Optical Society of America Meeting on Photon Correlation Techniques and Applications, May 1988.

3. EG\&G, Inc., Optoelectronics Division, Vaudreux, Quebec, Canada (formerly a division of RCA).

4. Paul Horowitz and Winfield Hill, The Art of Electronics, Cambridge University Press, 1990.

5. S. Aronson et al., DO design report, Fermilab, November 1984.

6. LeCroy Corporation, 700 Chestnut Ridge Road, Chestnut Ridge, NY, 1097\%, U.S.A.

7. AMP Corporation, Harrisburg, PA, 17105, U.S.A.

8. Philips Cumponents, 1755 North Collins Blvd., Richardson, TX, 75080, U.S.A.

9. Figure courtesy of R.J. McIntyre, EG\&G Optoelectronics. 

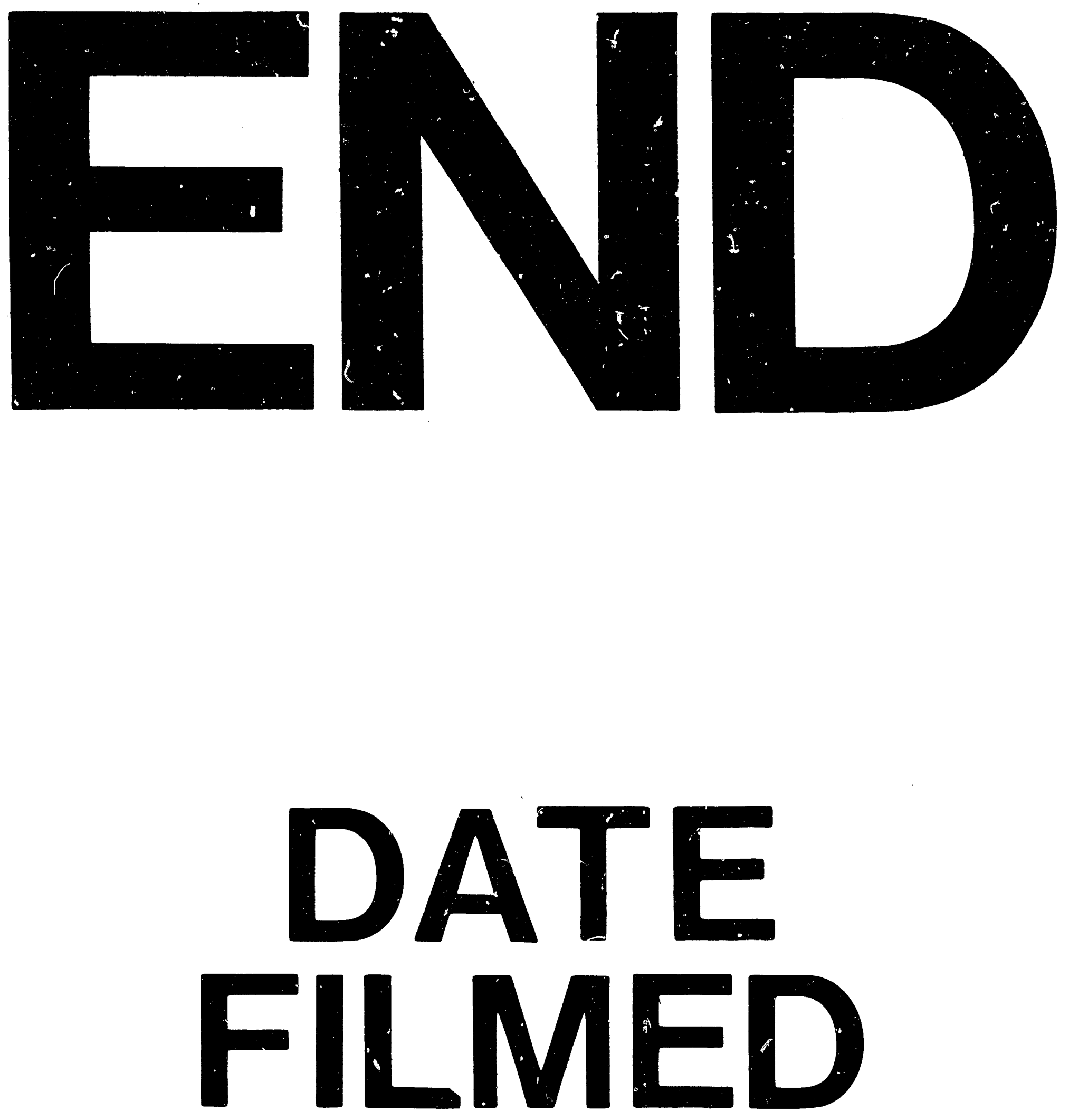

$-1$

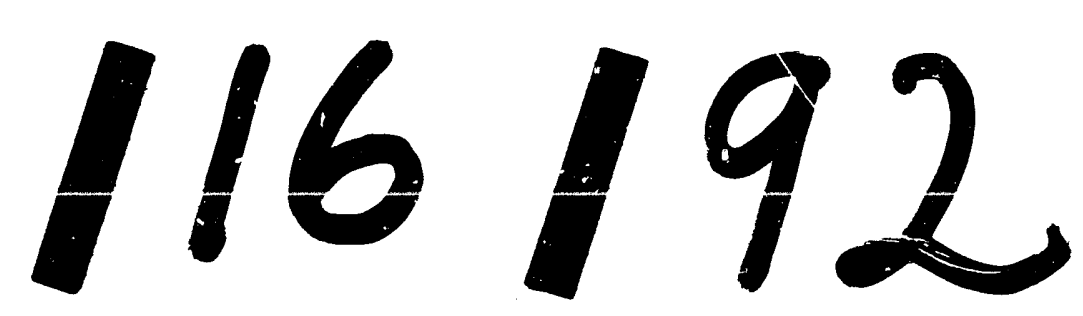


